\title{
Video Article \\ Surgical Procedures for a Rat Model of Partial Orthotopic Liver Transplantation with Hepatic Arterial Reconstruction
}

\author{
Kazuyuki Nagai ${ }^{1,2}$, Shintaro Yagi ${ }^{2}$, Shinji Uemoto ${ }^{2}$, Rene H. Tolba ${ }^{1}$ \\ ${ }^{1}$ Institute for Laboratory Animal Science and Experimental Surgery, RWTH-Aachen University \\ ${ }^{2}$ Department of Hepato-Biliary-Pancreatic Surgery and Transplantation, Graduate School of Medicine, Kyoto University
}

Correspondence to: Kazuyuki Nagai at kaznagai@kuhp.kyoto-u.ac.jp

URL: https://www.jove.com/video/4376

DOI: doi:10.3791/4376

Keywords: Medicine, Issue 73, Biomedical Engineering, Anatomy, Physiology, Immunology, Surgery, liver transplantation, liver, hepatic, partial, orthotopic, split, rat, graft, transplantation, microsurgery, procedure, clinical, technique, artery, arterialization, arterialized, anastomosis, reperfusion, rat, animal model

Date Published: $3 / 7 / 2013$

Citation: Nagai, K., Yagi, S., Uemoto, S., Tolba, R.H. Surgical Procedures for a Rat Model of Partial Orthotopic Liver Transplantation with Hepatic Arterial Reconstruction. J. Vis. Exp. (73), e4376, doi:10.3791/4376 (2013).

\section{Abstract}

Orthotopic liver transplantation (OLT) in rats using a whole or partial graft is an indispensable experimental model for transplantation research, such as studies on graft preservation and ischemia-reperfusion injury ${ }^{1,2}$, immunological responses ${ }^{3,4}$, hemodynamics ${ }^{5,6}$, and small-for-size syndrome ${ }^{7}$. The rat OLT is among the most difficult animal models in experimental surgery and demands advanced microsurgical skills that take a long time to learn. Consequently, the use of this model has been limited. Since the reliability and reproducibility of results are key components of the experiments in which such complex animal models are used, it is essential for surgeons who are involved in rat OLT to be trained in wellstandardized and sophisticated procedures for this model.

While various techniques and modifications of OLT in rats have been reported ${ }^{8}$ since the first model was described by Lee et al. ${ }^{9}$ in 1973 , the elimination of the hepatic arterial reconstruction ${ }^{10}$ and the introduction of the cuff anastomosis technique by Kamada et al. ${ }^{11}$ were a major advancement in this model, because they simplified the reconstruction procedures to a great degree. In the model by Kamada et al., the hepatic rearterialization was also eliminated. Since rats could survive without hepatic arterial flow after liver transplantation, there was considerable controversy over the value of hepatic arterialization. However, the physiological superiority of the arterialized model has been increasingly acknowledged, especially in terms of preserving the bile duct system ${ }^{8,12}$ and the liver integrity ${ }^{8,13,14}$.

In this article, we present detailed surgical procedures for a rat model of OLT with hepatic arterial reconstruction using a $50 \%$ partial graft after ex vivo liver resection. The reconstruction procedures for each vessel and the bile duct are performed by the following methods: a 7-0 polypropylene continuous suture for the supra- and infrahepatic vena cava; a cuff technique for the portal vein; and a stent technique for the hepatic artery and the bile duct.

\section{Video Link}

The video component of this article can be found at https://www.jove.com/video/4376/

Protocol

\section{Basic Techniques and Common Procedures}

1. All procedures are carried out under clean but nonsterile conditions.

2. Once the abdomen of the rat is opened, all procedures are performed under a surgical microscope at a magnification of 16x. Exceptions are the ex vivo liver resection, which is performed at $10 x$, and the following procedures, which are performed at $25 x$ : insertion of a stent into the bile duct and the hepatic artery, and the reconstruction of the hepatic artery, the infrahepatic vena cava (IHVC), and the bile duct.

3. Cotton swabs are used for gentle manipulation of organs, blunt dissection of tissues, and compression hemostasis. Gauze swabs (5 x $5 \mathrm{~cm}$ ) soaked with lactated Ringer solution are used to retract the liver or intestines, and to keep organs moist. The Satinsky clamp can be used for retraction of gauze-covered intestines toward the left or the tail of the rat to widen the surgical field around the IHVC.

4. All ligations are performed with a 6-0 silk thread except for the pedicles of liver lobes during ex vivo liver resection, where 4-0 silk threads are used. The ligature can be pulled by a DeBakey Bulldog clamp or other instruments to provide adequate tension to the ligated point so that the second ligature can be made at a distance to the first one, and the division between the two ligated points can be done properly.

5. All intravenous injections are performed through the penile vein.

6. During the reconstruction procedures for the suprahepatic vena cava (SHVC), the portal vein, the hepatic artery, and the bile duct, a small lump of oil-based clay is used to hold a finger ring of a peripheral vascular clamp or a mosquito forceps to keep them fixed in position. 


\section{Presurgical Preparation}

1. Male Lewis rats weighing between 230 and $250 \mathrm{~g}$ are used as the donor and recipient for liver transplantation. The rats are allowed free access to water and food until the induction of anesthesia.

2. Cuff for the portal vein (Figure 1): Prepare the cuff for the portal vein by cutting a 14-gauge catheter with a No. 11 scalpel blade under the microscope. The cuff consists of a body and an extension, each with a length of $2 \mathrm{~mm}$. Make circumferential grooves on the cuff by clamping the wall of the cuff step by step circumferentially with a mosquito forceps so that a thread can be fastened tightly on the cuff without slipping off.

3. Stents for the bile duct and hepatic artery (Figure 1): Cut 24-gauge catheters with a scalpel under the microscope to produce bevels at both ends of the catheters with a length of $4 \mathrm{~mm}$ for the hepatic artery and $5 \mathrm{~mm}$ for the bile duct.

\section{Donor Operation}

The schema of the removal of the liver from the donor rat is shown in Figure 2. This procedure requires approximately 30-35 min.

1. Anesthetize the rat with inhalation of $4 \mathrm{vol} \%$ isoflurane in $100 \%$ oxygen at a flow rate of $4 \mathrm{~L} / \mathrm{min}$ for the induction of the anesthesia, and 1.5 vol\% at $2 \mathrm{~L} / \mathrm{min}$ for the maintenance. Inject buprenorphine $(0.1 \mathrm{mg} / \mathrm{kg})$ subcutaneously as an analgesic. Place the rat on a heating pad, and fix the upper arms using the magnetic fixator retraction system (Figure 3a). Shave the fur from the entire abdominal area of the rat, and sterilize the corresponding skin with a povidone-iodine solution.

2. Open the abdomen by a midline incision with bilateral extensions. Place a 5-ml syringe under the back of the rat so that the SHVC is elevated ventrally. Using a mosquito forceps, clamp and pull the xiphoid process toward the head, and apply the subcostal retractors to open the surgical field (Figure 3a).

3. Dissect the falciform ligament and left triangular ligament. Next, ligate and divide the left phrenic vein.

4. Retract the median and left lateral lobes upward with a wet gauze swab. Using bipolar forceps, coagulate and divide the para-esophageal vessels between the left lateral and anterior caudate lobe.

5. Move the intestines outside the abdomen onto the left side of the rat, and cover them with a wet gauze swab. Retract the right lateral lobe upward with a wet gauze swab. Isolate the IHVC from the retroperitoneal tissue, and ligate the right adrenal vein, which will be divided later just before the graft removal.

6. To insert a stent into the bile duct (Figure $3 b$ ):

a. Ligate the bile duct at the level of the branching of the gastroduodenal artery. The soft tissues surrounding the bile duct should be preserved as much as possible, and the separation of the bile duct from the hepatic artery should be avoided to secure sufficient arterial blood supply to the bile duct.

b. With straight micro scissors, make a small incision in the anterior wall of the bile duct proximal to the ligated point. While holding the anterior wall of the incision with a straight micro forceps in the left hand, insert a stent into the duct using a curved micro forceps in the right hand, and secure it with a 6-0 silk thread. One of the cut ends of the thread on the bile duct is kept at a length of $4 \mathrm{~mm}$ so that the thread can be held during the later anastomosis.

7. Liberate the portal vein from the pyloric and splenic veins by ligating and dividing them.

8. Ligate and divide the gastroduodenal artery, then isolate the common hepatic artery (CHA) from the pancreatic head to its root. Rotate the liver to the right with cotton swabs, and dissect the ligament around the back of the liver and esophagus.

9. At the completion of the preparations for liver excision, remove the retractors, the mosquito forceps for the xiphoid process, and the 5-ml syringe under the back of the rat. Return the intestines to the abdominal cavity.

10. Inject $500 \mathrm{IU}$ of Heparin-Natrium in $2 \mathrm{ml}$ of normal saline solution through the penile vein. About $3 \mathrm{~min}$ later, reset the 5 -ml syringe, the mosquito forceps, and the retractors. Ligate the CHA proximal to its root. Keep one of the cut ends of the thread ligated for the CHA long.

11. After clamping the IHVC close to the right renal vein with a mosquito forceps, clamp the portal vein with a disposable micro vessel clamp below the stump of the splenic vein. Incise the anterior wall of the portal vein, and insert an 18-gauge catheter into the portal vein.

12. Perfuse the liver in situ with $60 \mathrm{ml}$ of cold histidine-tryptophan-ketoglutarate $(\mathrm{HTK})$ solution at a hydrostatic pressure of $20 \mathrm{~cm} \mathrm{H}_{2} \mathrm{O}$. Immediately afterward, cut the diaphragm and transect the intrathoracic vena cava, and cut the anterior wall of the IHVC open to allow the perfusion solution to be rinsed out of the liver (Figure 3c).

13. Clamp the IHVC with a disposable micro vessel clamp just below the liver. Excise the liver by dissecting the IHVC slightly below the middle point between the liver and the right renal vein, the portal vein below the stump of the splenic vein, the diaphragm, the remaining ligaments at the back of the liver, the right adrenal vein, and the $\mathrm{CHA}$ at its root. Place the excised liver in cold HTK solution in a metal cup mounted in a plastic box full of crushed ice.

\section{Ex vivo Graft Preparation}

All procedures for the liver graft are performed in the metal cup filled with ice-cold HTK solution. The ex vivo graft preparation requires approximately $30 \mathrm{~min}$.

1. For the attachment of a cuff to the portal vein (Figure 4):

Clamp the portal venous trunk with a DeBakey Bulldog clamp. Place the clamp in a bridging position over the cup (Figure 4a, b). Put the portal vein through the cuff, and clamp the portal vein again together with the extension of the cuff at the 12 o'clock position (Figure 4c). Evert the wall of the portal vein over the cuff to position the stump of the splenic vein outside of the cuff at the 7 o'clock position (Figure 4d), and secure the portal vein with a 6-0 silk thread (Figure 4e).

2. To insert a stent into the hepatic artery (Figure 5):

Fix the liver by clamping both edges of the diaphragm by forceps, and pull the CHA straight by holding the ligated thread with the DeBakey Bulldog clamp (Figure 5a). With straight micro scissors, make a small incision in the anterior wall of the CHA. With the left hand, hold the anterior wall of the incision with a straight micro forceps, and with the right hand, insert a stent into the $\mathrm{CHA}$ using a curved micro forceps. 
The stent is previously washed with Heparin-Natrium solution (100 IU/ml) (Figure $5 \mathbf{b}-\mathbf{d}$ ). Secure the stent with a $6-0$ silk thread, and keep one of the cut ends of the thread at a length of $4 \mathrm{~mm}$. Flush the liver through the arterial catheter with $5 \mathrm{ml}$ of cold HTK solution.

3. For $50 \%$ liver resection (Figure 6 ):

a. Clamp the posterior caudate lobe with a mosquito forceps to fix it in place. Resect the lobe after ligation of its pedicle with a 4-0 silk thread (Figure 7a). In the same manner, remove the anterior caudate lobe.

b. Rotate the plastic box 90 degrees. Clamp the right edge of the diaphragm and the left portion of the median lobe. Make a small incision at the upper edge of the border of the bilateral portions of the median lobe, and then remove the left portion after ligation (Figure $7 \mathrm{~b}$ ).

Remove the left lateral lobe after ligation of the pedicle with a 4-0 silk thread. Cauterize the resected liver surface carefully with bipolar forceps. As a result, the liver mass is reduced by approximately $50 \%{ }^{15}$ (Figure 7 c, d).

4. For plasty of the SHVC (Figure 8):

Fix the position of the liver by clamping both edges of the diaphragm with a mosquito forceps (Figure 8a). Trim the anterior wall of the SHVC by removing the corresponding diaphragm. Attach two 7-0 polypropylene sutures from the outside to the inside at both corners as stay sutures for the later anastomosis (Figure $\mathbf{8 b}$ ). Then trim the posterior wall of the SHVC.

5. Store the liver graft at $4{ }^{\circ} \mathrm{C}$ in HTK solution in a cold-water bath.

\section{Recipient Operation}

The schema of the graft implantation in the recipient rat is shown in Figure 9. The recipient operation requires 60-70 min, which includes $10-11$ min of anhepatic time and approximately $23-24$ min of IHVC clamping time.

1. Perform the same procedures as in the donor operation (3.1 to 3.4) except for the opening of the abdomen by a midline incision, without bilateral extensions (Figure 10a).

2. Place a wet gauze swab over the right side of the duodenum and whole intestines to obtain a surgical field around the IHVC. Put the left lateral and median lobes into the left subphrenic cavity, and retract the right lateral lobe upward with a wet gauze swab. Isolate the IHVC from the retroperitoneal tissue. Ligate and divide the right adrenal vein (Figure 10b). With a wet gauze and cotton swabs, rotate the liver to the left, and dissect the ligament around the back of the liver.

3. Return the right lateral lobe to the anatomical position. Place a wet gauze swab to cover and retract the median and left lateral lobes upward. Transect the bile duct just below the branch from the caudate lobe. The soft tissues surrounding the bile duct should be preserved as much as possible. Keep one of the cut ends of the thread ligated for the bile duct at 4-mm long.

4. Ligate and divide the gastroduodenal artery and the proper hepatic artery at a 3-mm distance to the branching from the $\mathrm{CHA}$. Then, make a Y-structure of the artery at the end of the CHA. Keep one of the cut ends of the thread ligated for the proper hepatic artery at 4-mm long. Rotate the liver to the right with cotton swabs, and dissect the ligament around the back of the liver from the left side.

5. After the intravenous injection of $2 \mathrm{ml}$ of lactated Ringer solution, clamp the IHVC with a metal micro vessel clamp just above the right renal vein. Clamp the portal vein at the level of its bifurcation in the liver hilum by a mosquito forceps from the left side of the rat. Clamp the SHVC together with the diaphragm from the right side by a peripheral vascular clamp, and fix the finger ring of the clamp in a lump of oil-based clay.

6. Reduce the anesthesia with isoflurane to 0.4 vol\% during the anhepatic time (the duration of cross-clamping of the portal vein). Excise the recipient native liver by dissecting the SHVC, the portal vein, and the IHVC at the following levels: the SHVC, at the border between the SHVC and the liver; and the portal vein, just above the jaw of the mosquito forceps; and the IHVC, slightly below the middle point between the liver and the right renal vein (Figure 10c). Place the liver graft orthotopically.

7. For the anastomosis of the SHVC by a continuous suture (Figure 11):

a. Use a curved micro forceps in the left hand during the suturing procedure for grasping the vessel wall or holding the suturing needle. First, place the stay suture on the recipient SHVC from the inside to the outside by using the attached 7-0 polypropylene at the right corner of the graft followed by tying a knot (or you can tie it after all suturing procedures are complete). Next, place the second stay suture in the same way at the left corner, which will be the starting stitch of a running suture. To widen the anastomosis, grasp and maintain the sutures using DeBakey bulldog clamps at both corners with gentle traction superiolaterally (Figure 11a, b).

b. Pierce the suture at the left corner through the wall on the graft side from the outside to the inside closely to the knot outside, and suture the posterior row of the SHVC intraluminally with 7 to 8 stitches to the right corner (Figure 11c). Make the first few stitches carefully so that the inside lumens are faced each other. At the right corner, pierce the 7-0 polypropylene through the vessel on the graft side to the outside.

c. Next, suture the anterior row from the outside, from right to left, with approximately 10 stitches (Figure 11d). Before the completion of the anterior row, flush the inside with lactated Ringer solution to remove air bubbles. Make the last stitch at the anterior row as close as possible to the stay suture at the left corner, and then tie them together.

8. For the reconstruction of the portal vein by a cuff technique (Figure 12):

a. Retract the median and left lateral lobes upward with a wet gauze swab. Clamp the recipient portal vein at its confluence with the pyloric vein by using a disposable micro vessel clamp from the right. Fix the mosquito forceps that clamps the portal vein in the clay, and pull the tip of the forceps toward the liver hilum (Figure 12a, b).

b. Incise the anterior wall of the portal vein just below the jaw of the mosquito forceps. Wash the inside of the recipient portal vein and the cuff with lactated Ringer solution. Hold the anterior wall of the incision with a straight micro forceps in the left hand and an extension of the cuff with a curved micro forceps in the right hand. Insert the cuff into the recipient portal vein deeply, and secure it with a circumferential 6-0 silk thread (Figure 12c-f).

c. Release the clamps of the portal vein and SHVC, and then reperfuse the liver. Remove the 5-ml syringe from the back of the rat, and increase the concentration of isoflurane to $0.8 \mathrm{vol} \%$.

9. For the reconstruction of the hepatic artery by a stent technique ${ }^{16}$ (Figure 13):

a. First, hold the thread of the recipient proper hepatic artery by a mosquito forceps from the left side, and pull it toward the liver hilum, and then clamp the recipient CHA from the right side close to the pancreas (Figure 13a).

b. With a straight micro forceps, make a small incision in the bifurcation of the Y-structure at the end of the recipient $\mathrm{CHA}$ to make a funnel-shaped opening. Hold the stent placed in the graft $\mathrm{CHA}$ with a curved micro forceps. After washing each lumen with Heparin- 
Natrium solution $(100 \mathrm{IU} / \mathrm{ml})$, slide the stent into the recipient $\mathrm{CHA}$ and secure it with a 6-0 silk thread. Tie one end of this thread on the recipient $\mathrm{CHA}$ and the 4-mm thread on the graft $\mathrm{CHA}$ together so that both $\mathrm{CHAs}$ get closer to each other with reduced tension of the anastomotic site (Figure 13b). After that, release the clamp.

10. For the anastomosis of the IHVC by a continuous suture (Figure 14):

Anastomose in the same fashion as for the SHVC, but use more stitches with finer bite (Figure 14a-d). Tying of the sutures at the last stitch can be eliminated, or tying can be accomplished with a growth factor to avoid the anastomotic stricture caused by tying too tightly. After declamping, increase the concentration of anesthesia to $1.0 \mathrm{vol} \%$. If the anastomosis looks stenotic, dilate the anastomotic site by pulling the bilateral stay sutures or widening the anterior row gently to expand the anastomosis.

11. Administer $0.5 \mathrm{ml}$ of $8.4 \%$ sodium bicarbonate solution with $1.0 \mathrm{ml}$ of lactated Ringer solution intravenously.

12. Apply small fragments of Tachosil to seal the resected liver surface to prevent bleeding and biliary leakage.

13. For the reconstruction of the bile duct by a stent technique:

a. Hold the thread of the recipient bile duct by a mosquito forceps from the left side. Fix the mosquito forceps in the clay, and pull the tip of the forceps toward the liver hilum.

b. Make a small incision in the bile duct at the appropriate level so that the reconstructed bile duct would not be too long. Insert the stent placed in the graft bile duct into the recipient duct with careful attention to avoid a twist, and secure it with a 6-0 silk thread. Tie this thread on the recipient duct and the 4-mm thread on the graft duct together so that both ducts get closer to each other with reduced tension of the anastomotic site.

14. At the completion of the reconstruction procedures, inject $1 \mathrm{ml}$ of $5 \%$ glucose solution intravenously (Figure 15).

15. Confirm adequate hemostasis, and then close the abdominal incision by continuous 4-0 Vicryl sutures in two layers.

\section{Postoperative Treatment and Follow-up}

Immediately after the operation, treat the recipient rat with a subcutaneous injection of cefuroxime sodium (16 mg/kg) and buprenorphine (0.1 $\mathrm{mg} / \mathrm{kg}$ ) in a total of $1.5 \mathrm{ml}$ of normal saline solution. Allow the rat to recover for $60 \mathrm{~min}$ in a special intensive care unit cage with warmed air $\left(30-35^{\circ} \mathrm{C}\right)$ and an oxygen supply. Inject buprenorphine $(0.1 \mathrm{mg} / \mathrm{kg})$ subcutaneously as an analgesic every 12 hours for 3 days. Afterward move the rat to a normal cage, and provide ad libitum access to water and food.

\section{Representative Results}

All recipient rats $(n=20)$ survived without apparent complications until planned euthanasia for blood sampling at 1, 3, 24, and 168 hr $(7$ days) after portal reperfusion ( $n=5$ at each time point). The blood samples were collected from the IHVC by a direct puncture with a 27-gauge needle. Following the centrifugation at 5,340 $\mathrm{g}$ for $10 \mathrm{~min}$, the serum samples were obtained and analyzed for alanine aminotransferase (ALT) levels, which reflect the degree of hepatocellular damage after transplantation. The time-course of changes in serum ALT levels is shown in Figure 16. The ALT levels reached a peak at $24 \mathrm{hr}$ (mean \pm standard deviation: $212.6 \pm 67.9 \mathrm{IU} / \mathrm{L}$ ) and then declined to within normal limits at $168 \mathrm{hr}(33.6 \pm$ $6.8 \mathrm{IU} / \mathrm{L})$.

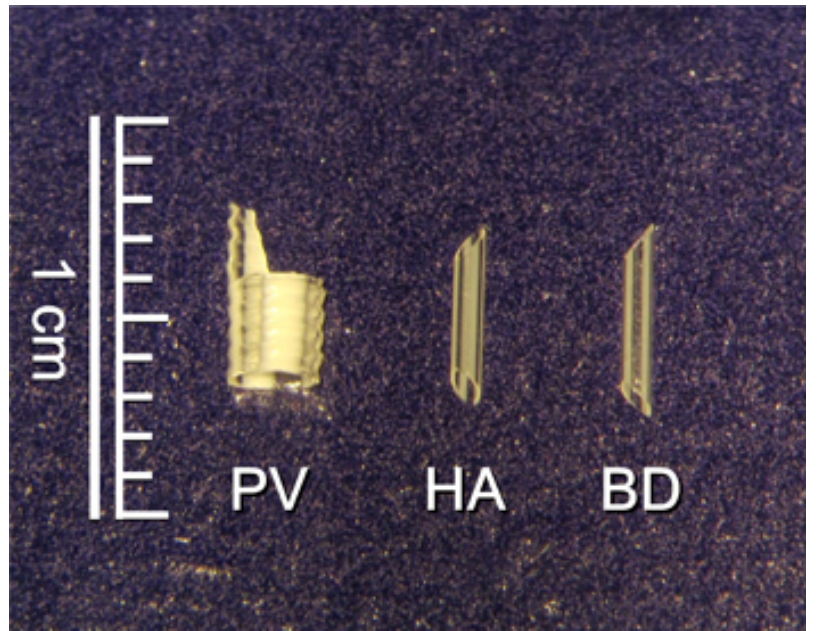

Figure 1. A cuff for the portal vein (PV) from a 14-gauge catheter, and stents for the hepatic artery (HA) and bile duct (BD) from 24-gauge catheters. 


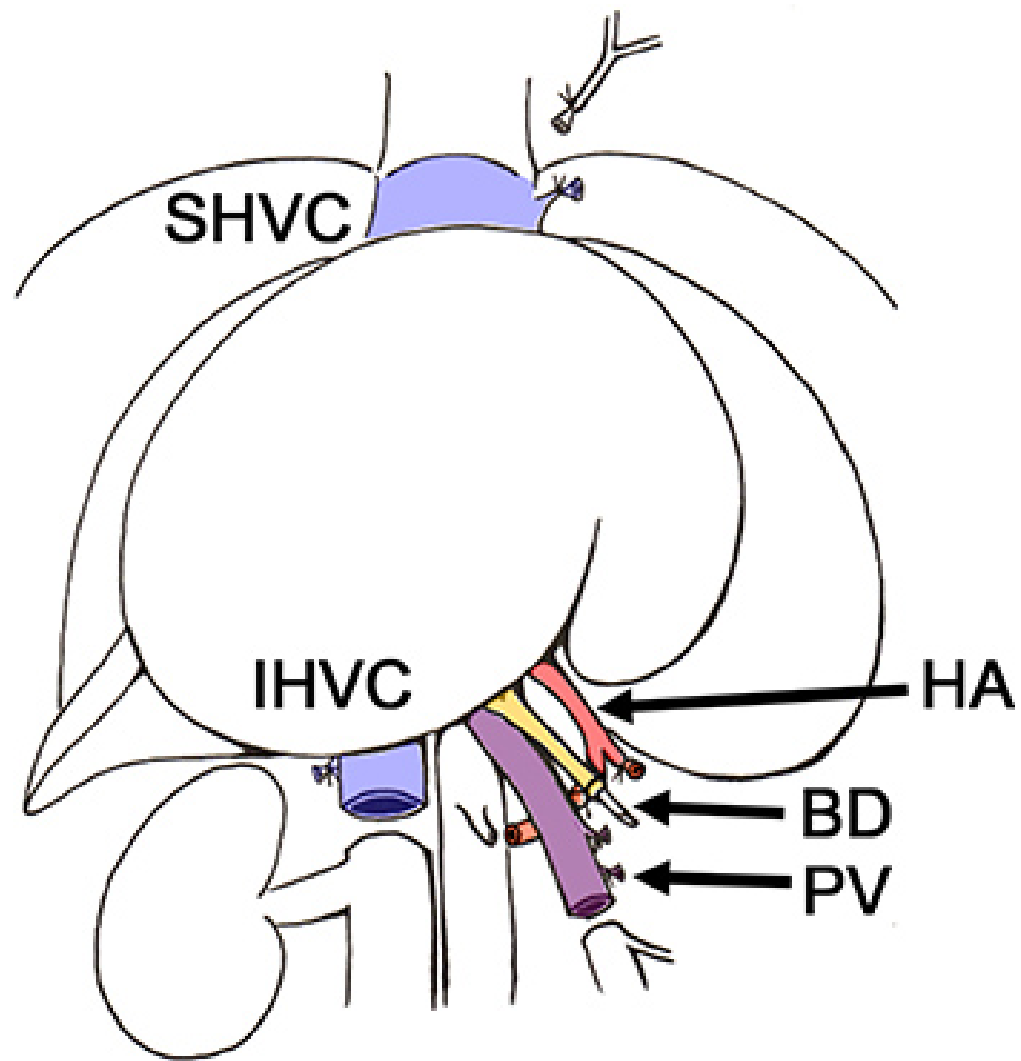

Figure 2. Schema of the removal of the liver from the donor rat. BD, bile duct; HA, hepatic artery; IHVC, infrahepatic vena cava; PV, portal vein; SHVC, suprahepatic vena cava.
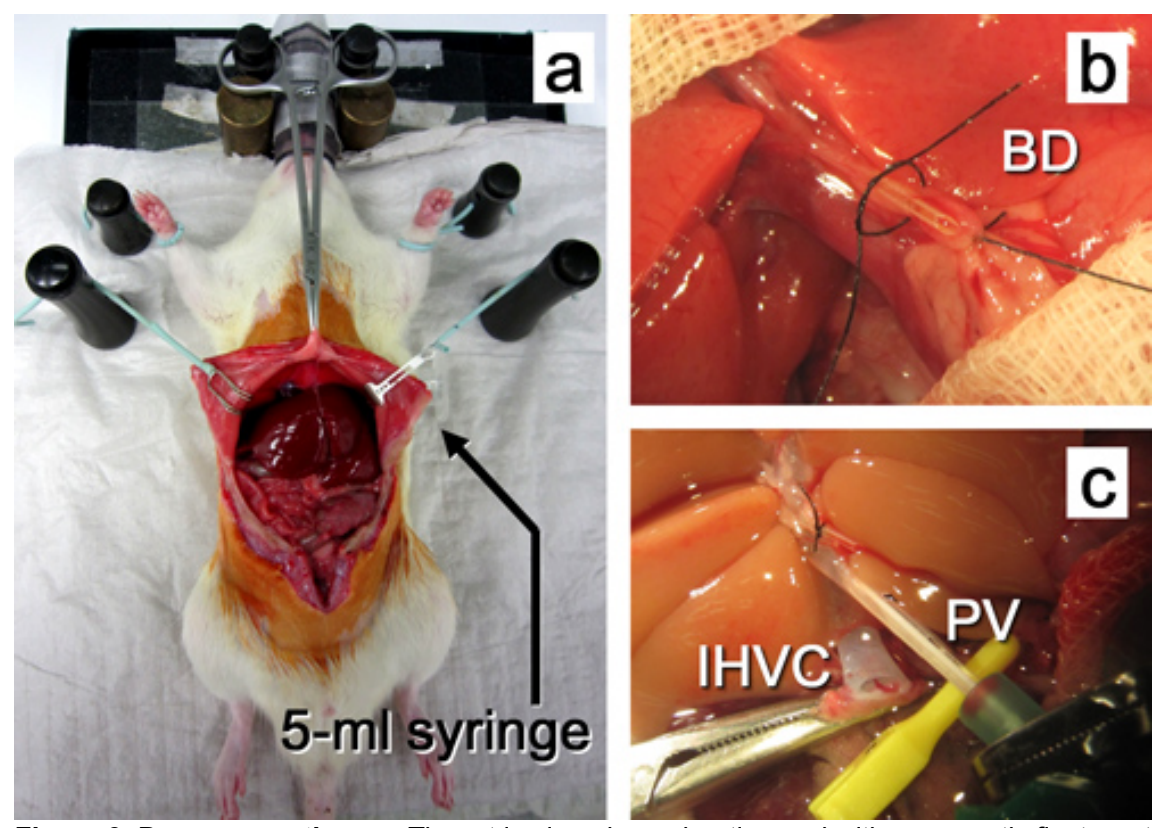

Figure 3. Donor operation. a. The rat is placed on a heating pad with a magnetic fixator retraction system. The abdomen is opened by a midline incision with bilateral extensions. b. Insertion of the stent into the bile duct. c. Perfusion of the liver through the portal vein. Abbreviations are explained in Figure 2. 


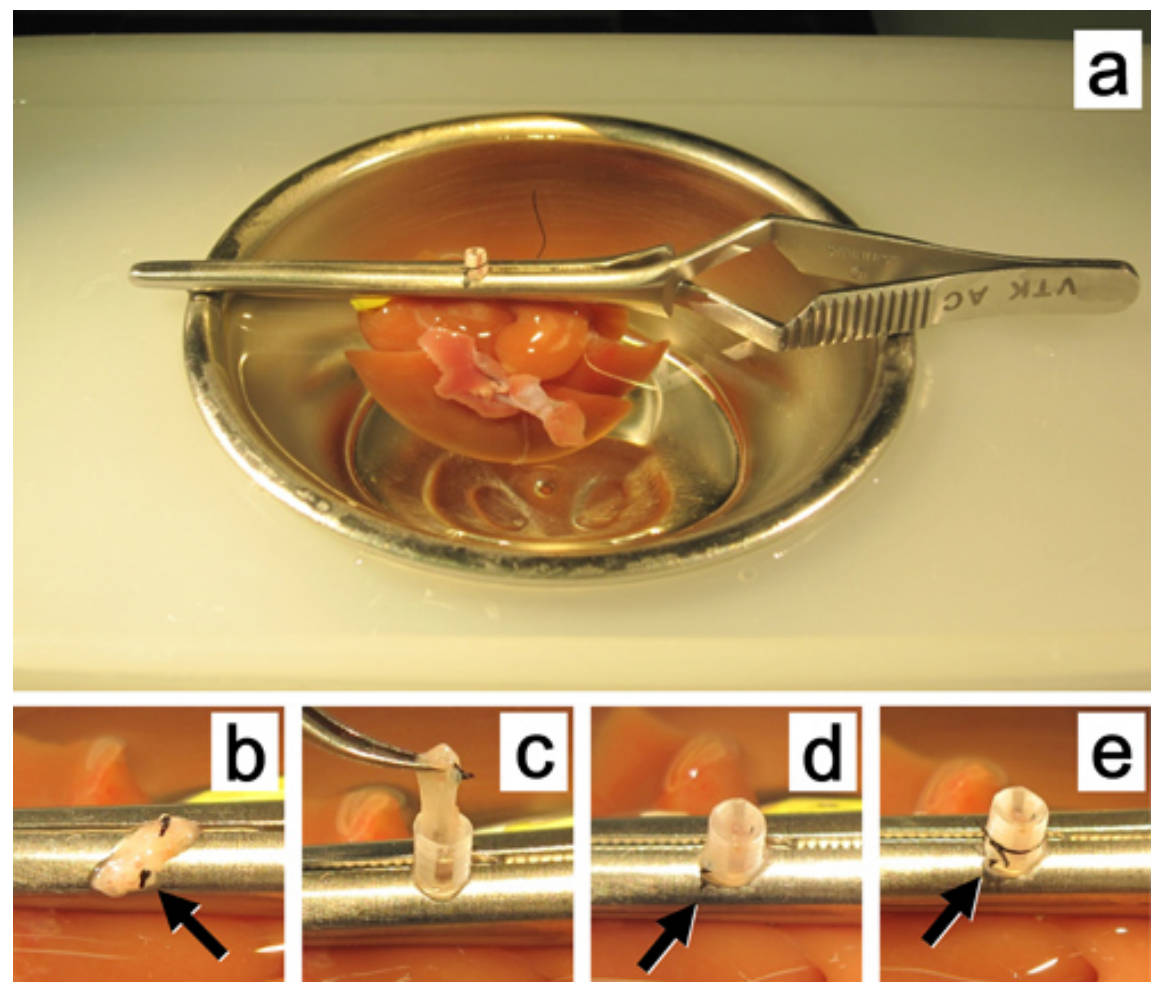

Figure 4. Attachment of a cuff to the portal vein. a,b. The DeBakey Bulldog clamp that grasps the portal venous trunk is placed over the metal cup. The cup is mounted in the plastic box filled with crushed ice. c. The portal vein is put through the cuff. $\mathbf{d}$. The wall of the portal vein is everted over the cuff with the stump of the splenic vein outside the cuff at the 7 o'clock position and an extension of the cuff at the 12 o'clock position. e. The portal vein is secured with a circumferential 6-0 silk thread on the cuff. The black arrows indicate the stump of the splenic vein.
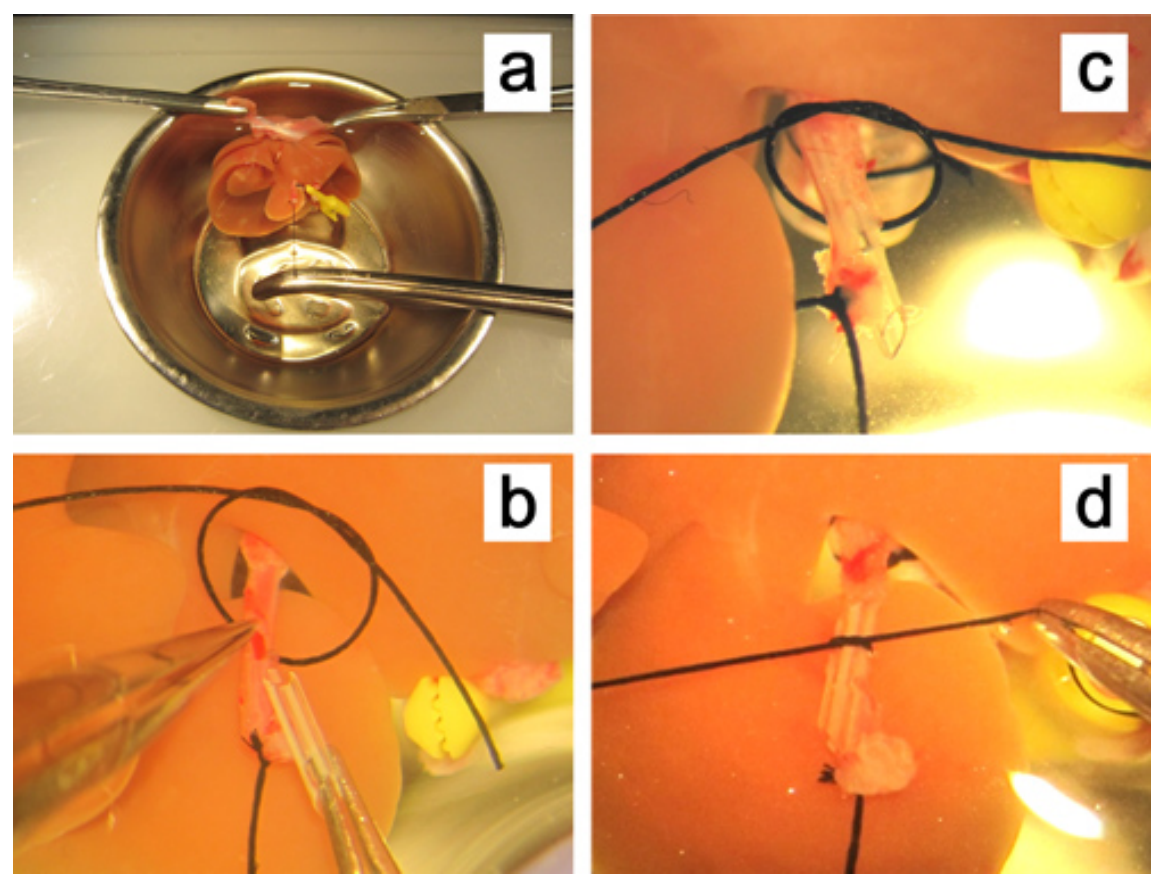

Figure 5. Ex vivo insertion of a stent into the hepatic artery. a. The liver is fixed by clamping both edges of the diaphragm, and the hepatic artery is pulled straight by holding the thread ligated for the artery. $\mathbf{b}$. The anterior wall of the small incision on the hepatic artery is held with a straight micro forceps. c, d. The stent is inserted into the hepatic artery and secured with a 6-0 silk thread. 


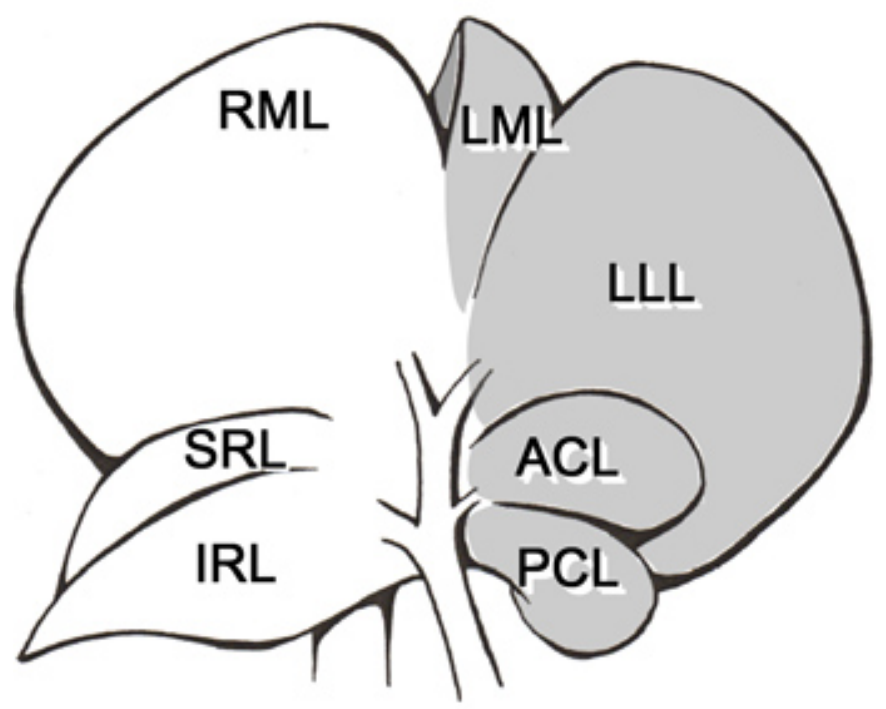

Figure 6. The schema of ex vivo $\mathbf{5 0 \%}$ liver resection. Lobes in gray color are removed. $\mathrm{ACL}$, anterior caudate lobe; $\mathrm{PCL}$, posterior caudate lobe; LLL, left lateral lobe; LML, left portion of the median lobe; RML, right portion of the median lobe; SRL, superior right lateral lobe; IRL, inferior right lateral lobe.
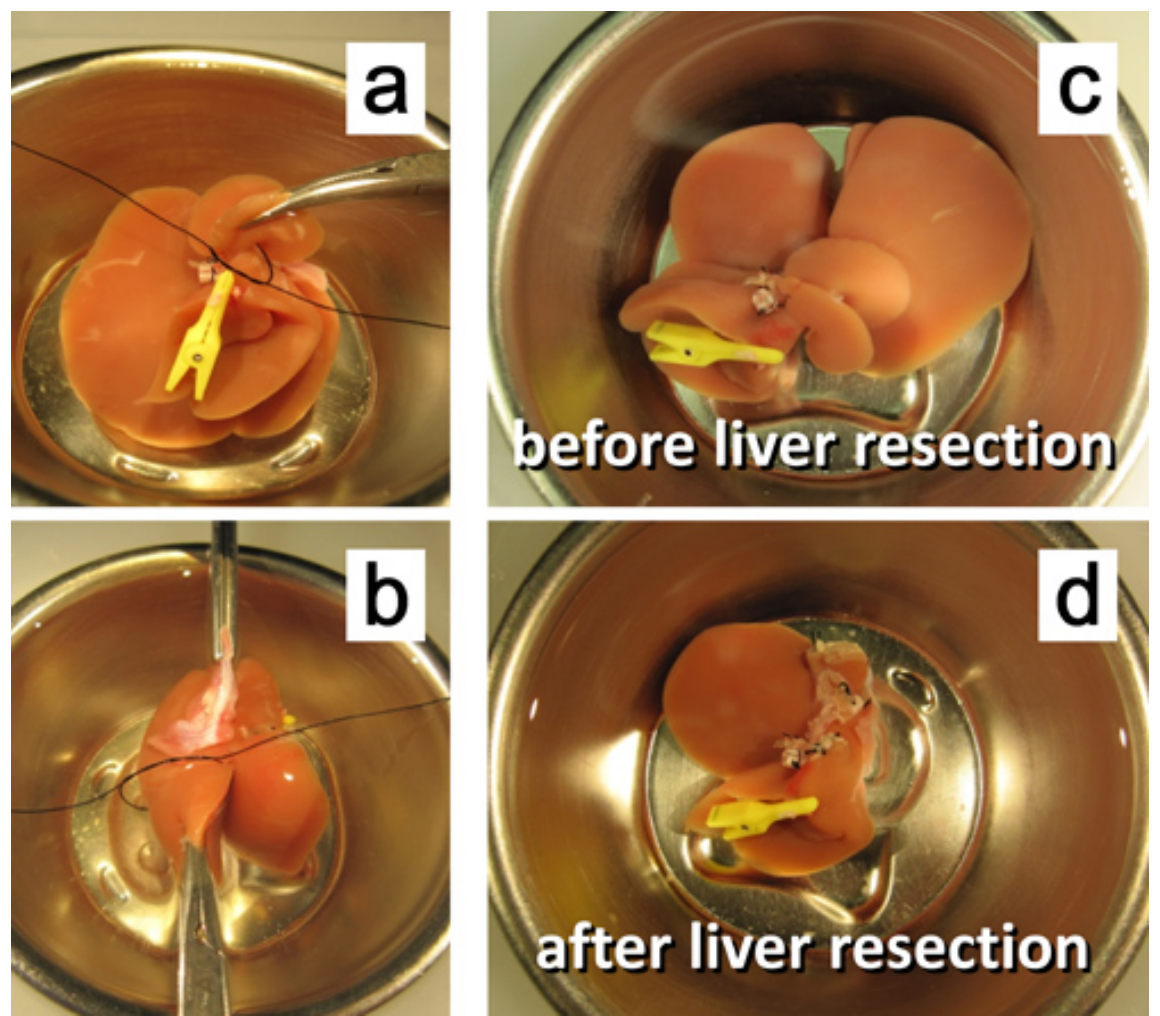

Figure 7. Ex vivo $\mathbf{5 0 \%}$ liver resection. a. Ligation of the pedicle of the posterior caudate lobe. $\mathbf{b}$. Ligation of the pedicle of the left portion of the median lobe. c. The liver before $50 \%$ resection. d. The liver after $50 \%$ resection. 


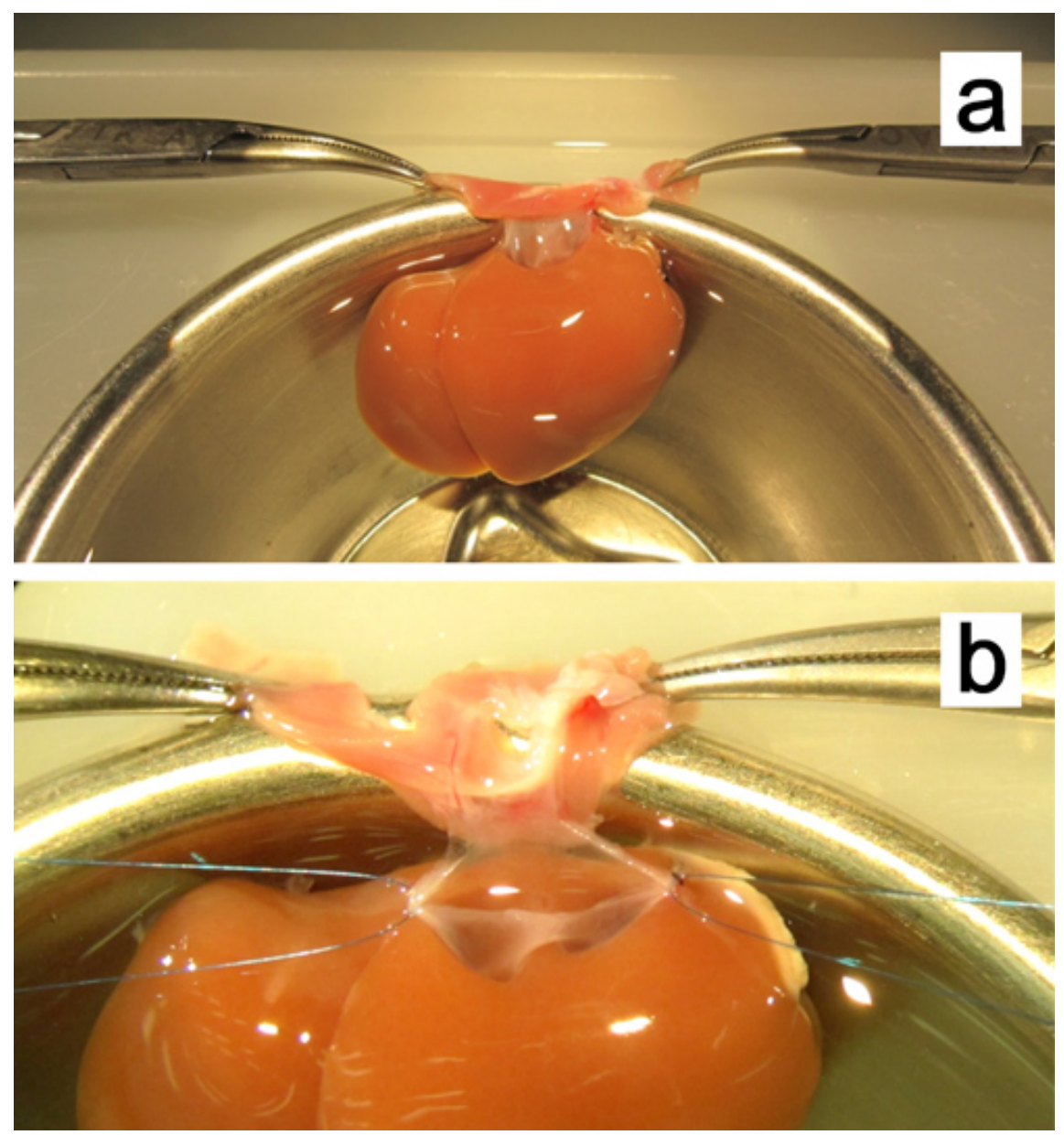

Figure 8. Ex vivo plasty of the suprahepatic vena cava. a. The liver is fixed by clamping both edges of the diaphragm with the mosquito forceps. b. Stay sutures with 7-0 polypropylene are attached at both corners. 


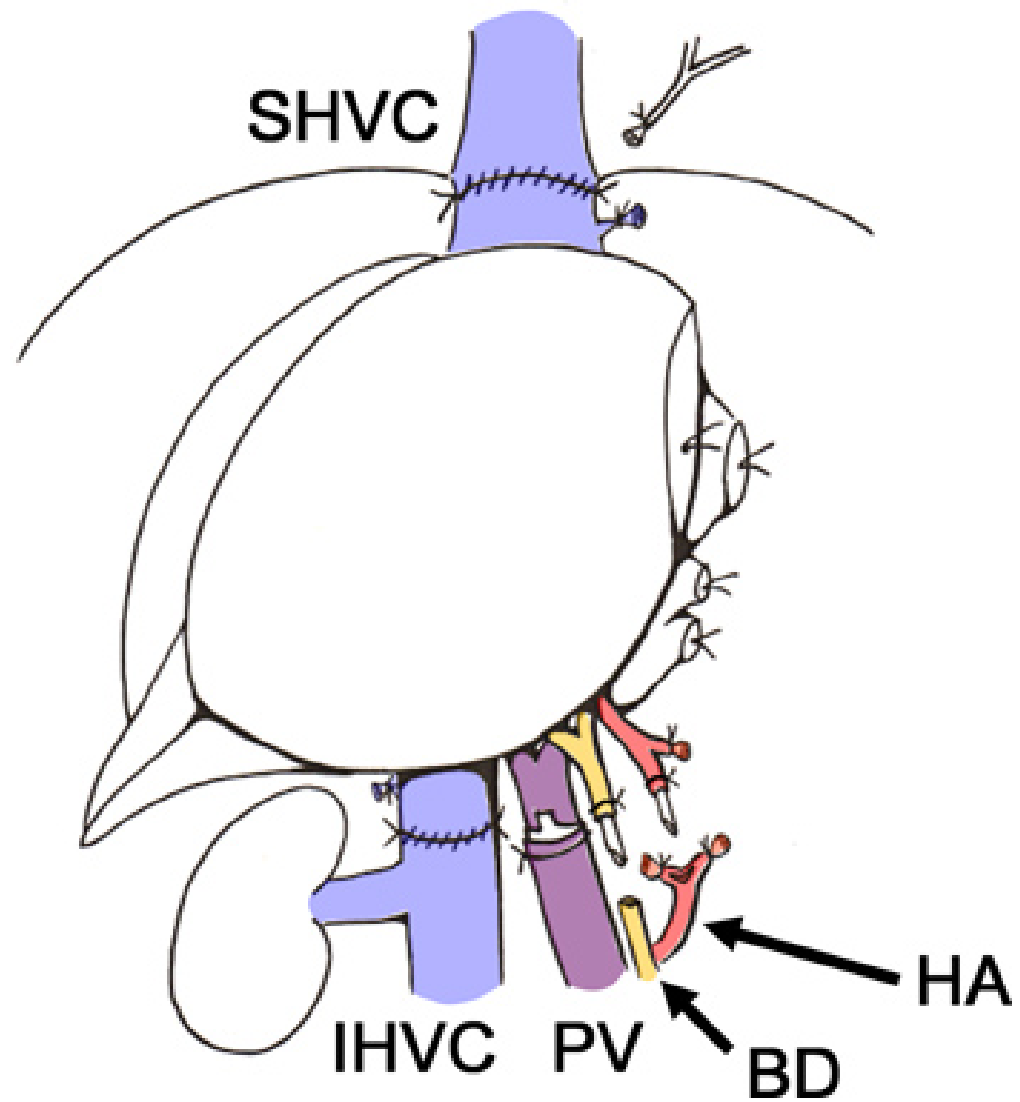

Figure 9. Schema of the graft implantation in the recipient rat. The reconstruction procedures are performed for the supra- and infrahepatic vena cava (SHVC and IHVC) with a 7-0 continuous suture, the portal vein (PV) by a cuff technique, and the hepatic artery (HA) and bile duct (BD) by a stent technique.
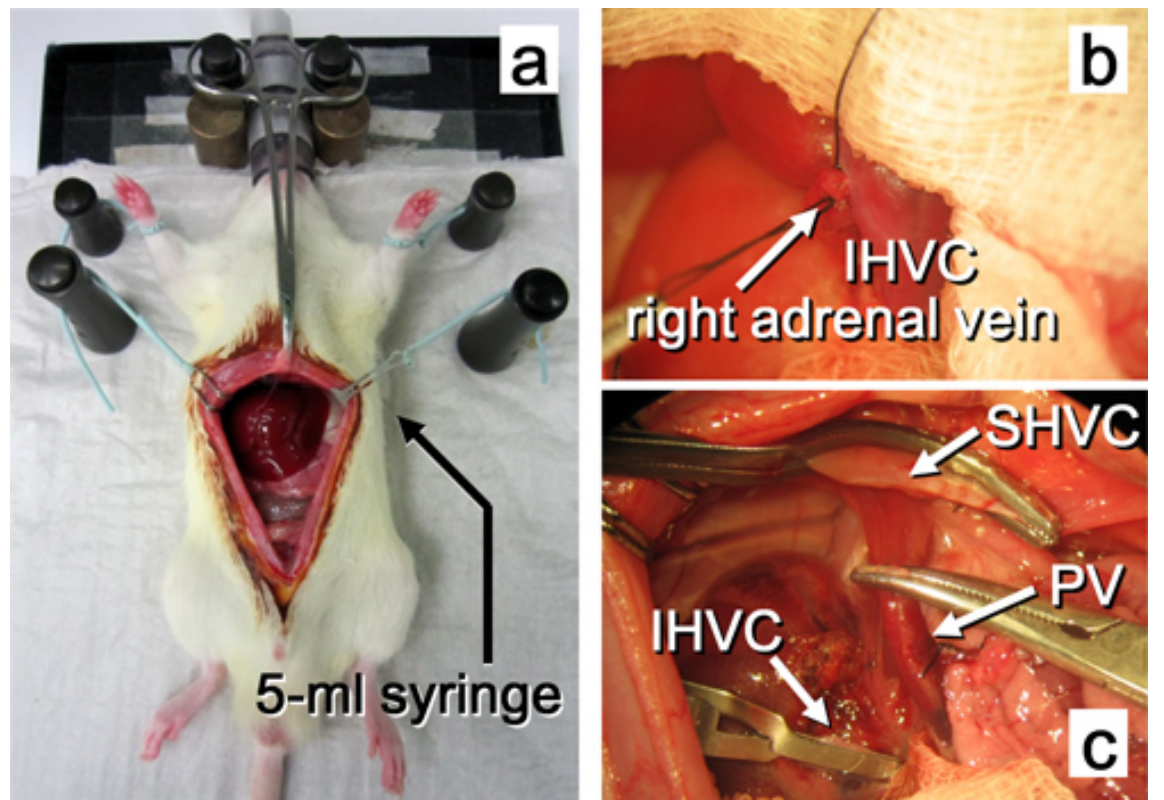

Figure 10. Recipient operation until the removal of the native liver. $\mathbf{a}$. The abdomen is opened by a midline incision. b. The right adrenal vein is ligated. c. The native liver is excised. Abbreviations are explained in Figure 2. 

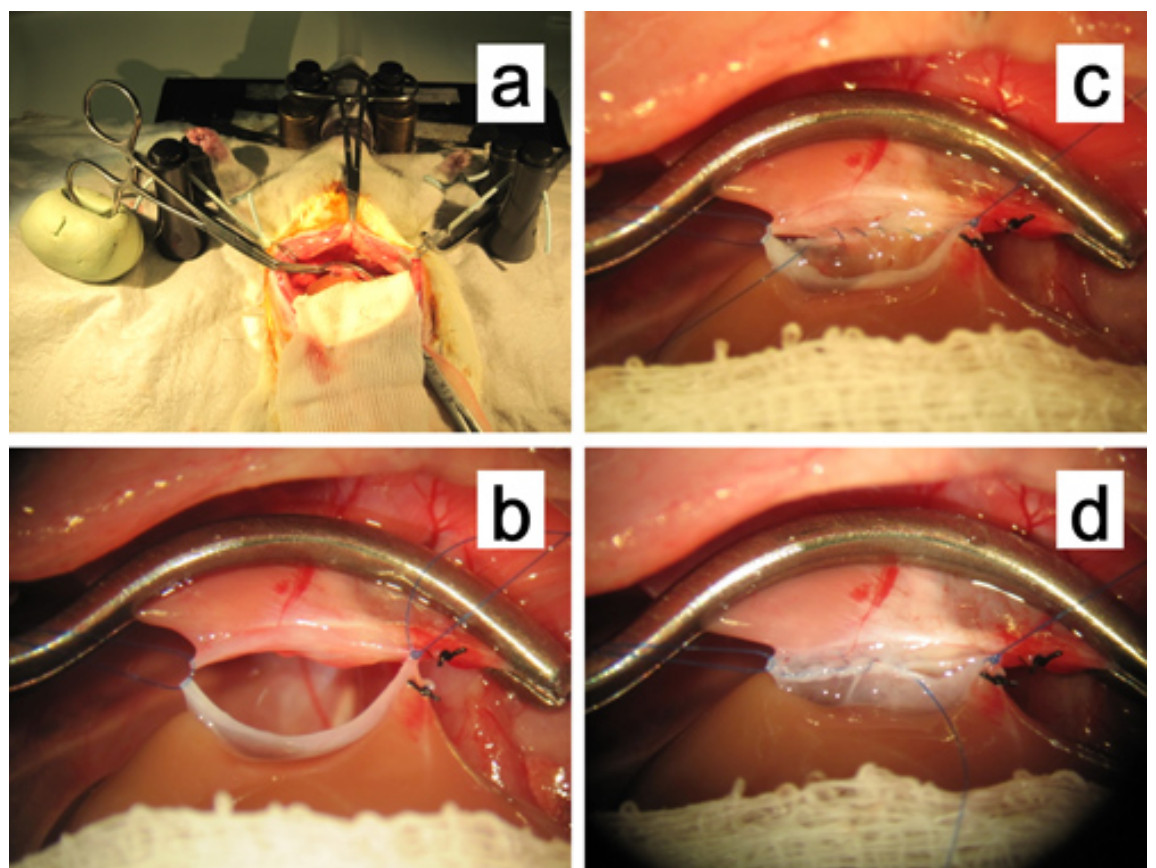

Figure 11. Anastomosis of the suprahepatic vena cava. a, b. The peripheral vascular clamp for the suprahepatic vena cava is fixed in a lump of oil-based clay. The stay sutures at both corners are maintained with gentle traction superiolaterally to widen the anastomosis. c. Continuous intraluminal suture of the posterior row in progress. $\mathbf{d}$. Continuous suture of the anterior row in progress. 

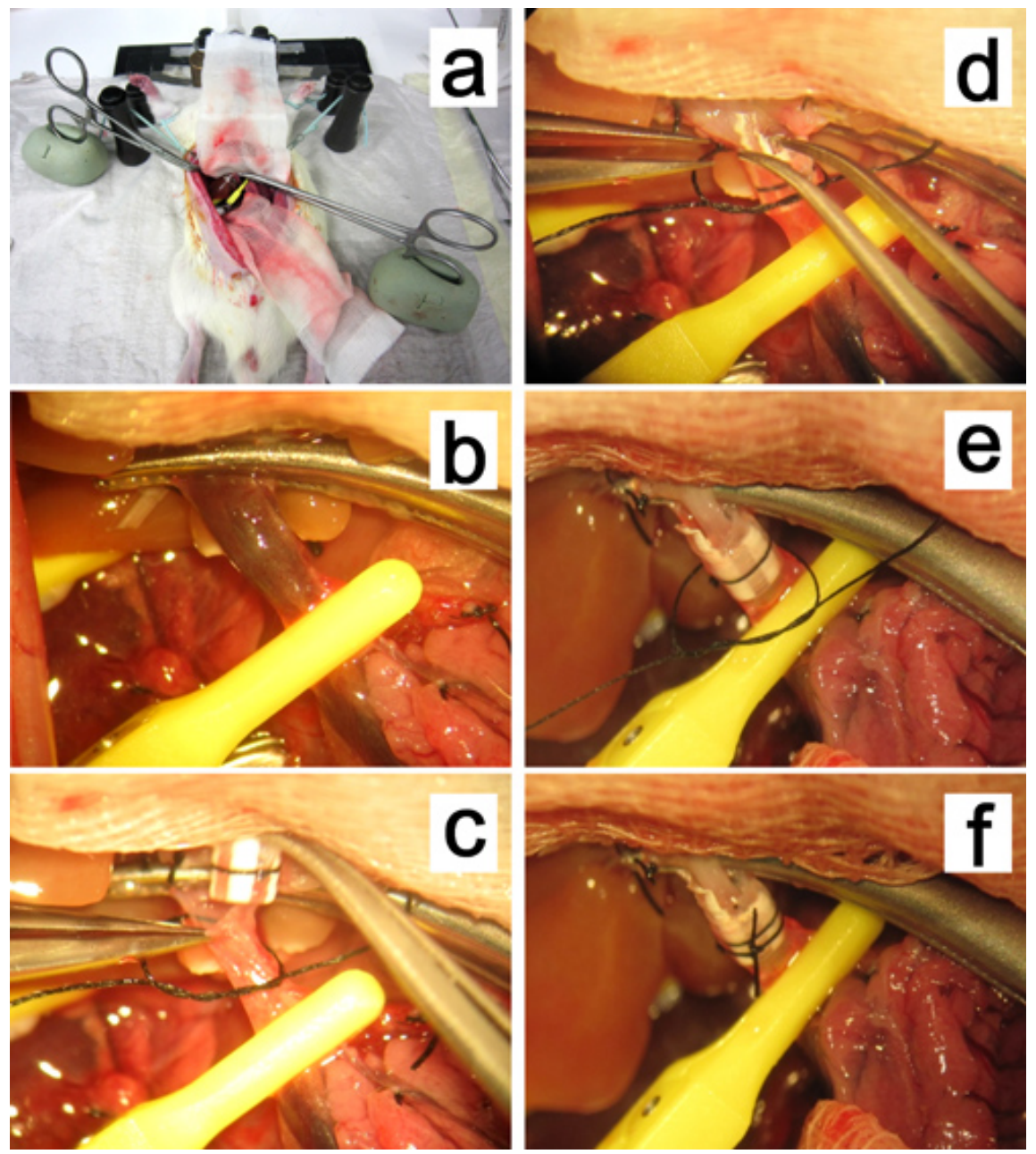

Figure 12. Reconstruction of the portal vein. a, b. The mosquito forceps clamping the portal vein is fixed in the oil-based clay and pulled toward the liver hilum. c-f. Insertion of the cuff into the portal vein.
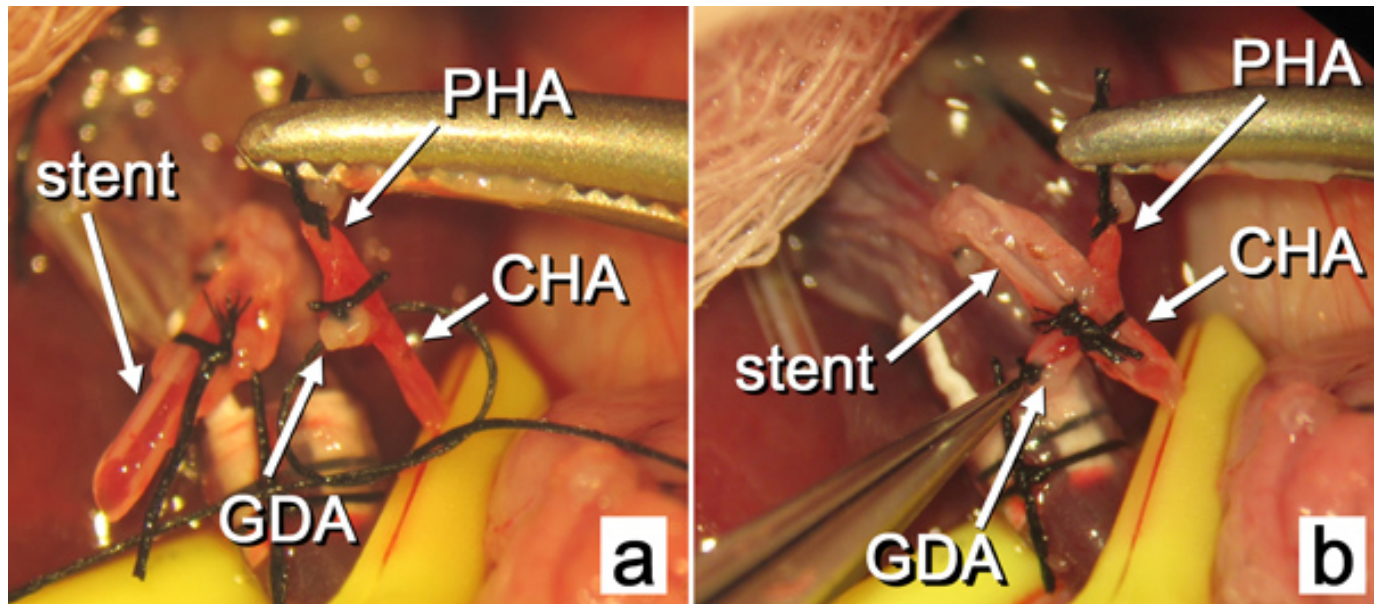

Figure 13. Reconstruction of the hepatic artery. a, b. Insertion of a stent into the recipient common hepatic artery (CHA) at the bifurcation of the proper hepatic artery (PHA) and gastroduodenal artery (GDA) 

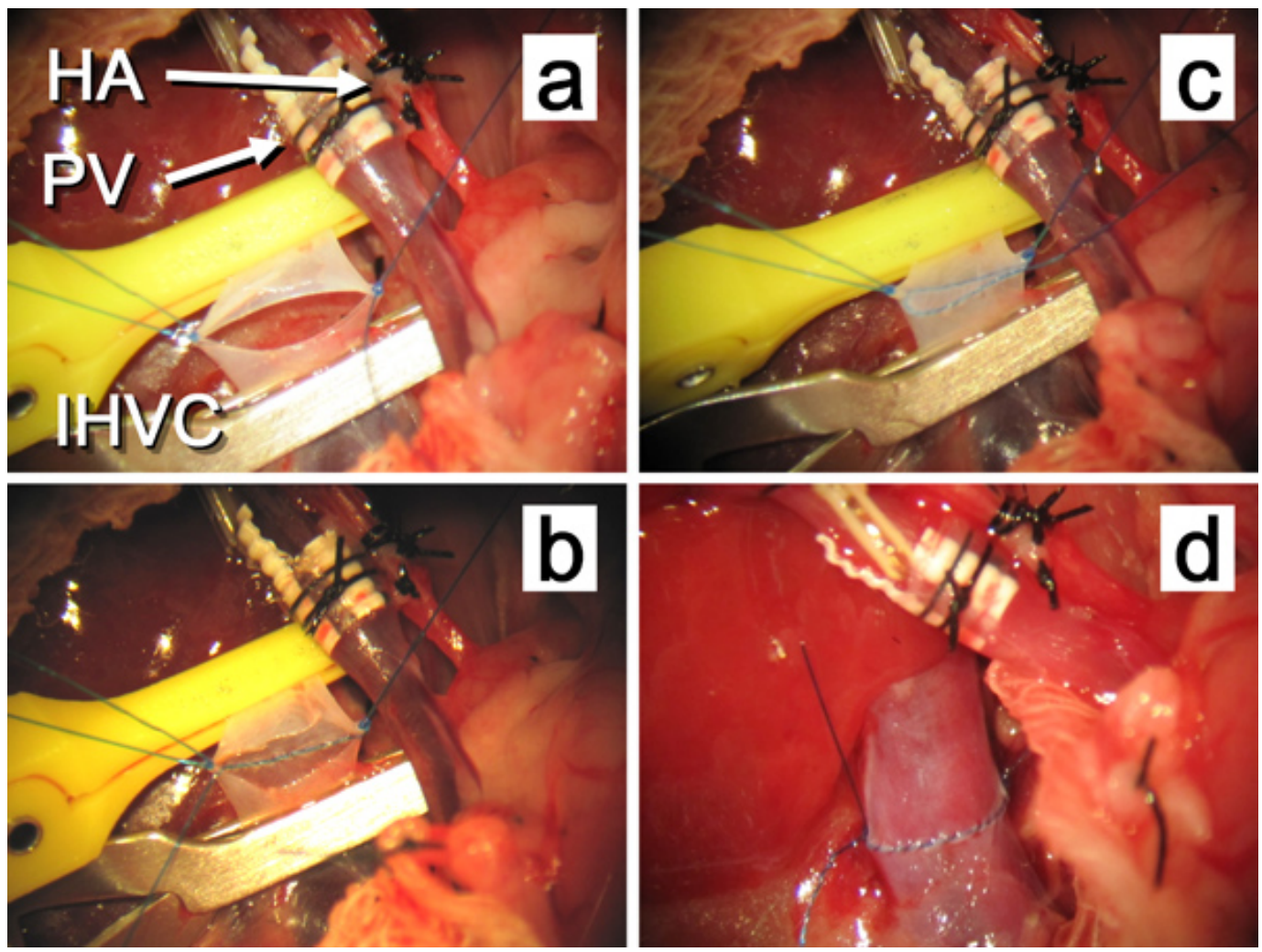

Figure 14. Anastomosis of the infrahepatic vena cava. a. The stay sutures at both corners. b. Continuous suture of the posterior row. c. Continuous suture of the anterior row. d. Reperfusion of the infrahepatic vena cava. Abbreviations are explained in Figure 2.

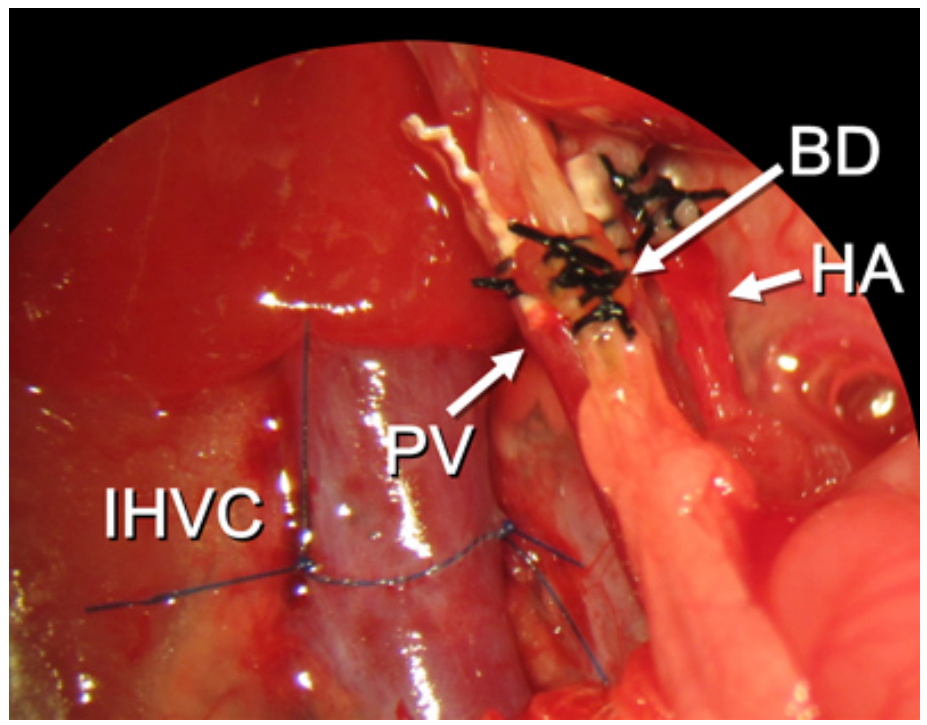

Figure 15. All the reconstruction procedures are finished. Abbreviations are explained in Figure 2. 


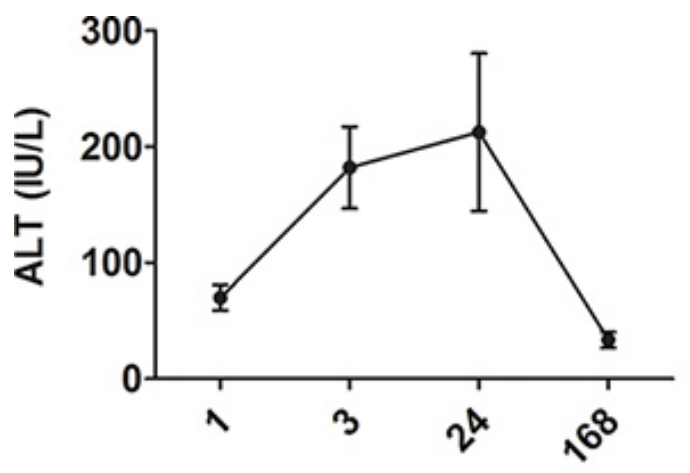

Time after portal reperfusion (hour)

Figure 16. Postoperative time-course of changes in serum alanine aminotransferase (ALT) levels ( $n=20 ; n=5$ at each time point). Data are expressed as means with error bars that indicate standard deviations. The ALT levels reached a peak at $24 \mathrm{hr}(212.6 \pm 67.9 \mathrm{IU} / \mathrm{L})$ and then declined to within normal limits at $168 \mathrm{hr}(33.6 \pm 6.8 \mathrm{IU} / \mathrm{L})$.

\section{Discussion}

The first model of rat OLT was reported by Lee et al. in $1973^{9}$, in which all vessels including the hepatic artery were reconstructed by a handsewn method and the extracorporeal portosystemic shunt was used. This model was technically complicated and difficult to perform. The next model was one without hepatic arterial reconstruction and the extracorporeal shunt, developed by the same authors ${ }^{10}$ in 1975 . Subsequently in 1979, Kamada et al. introduced the cuff anastomosis technique for the model without hepatic rearterialization ${ }^{11}$. With these modifications, OLT in rats was simplified with a shortened anhepatic time in recipient operations and has been widely used as an accepted experimental model.

However, there has been considerable controversy since then over the significance of hepatic arterialization in rat OLT ${ }^{8}$ because the arterialization was a demanding task but did not affect survival after transplantation. Numerous studies on hepatic arterialization using various reconstruction techniques have been reported ${ }^{8}$, such as an aortic segment-to-aorta anastomosis ${ }^{3,9,17}$, a cuff anastomosis technique ${ }^{18,19,20}$, a telescoping technique ${ }^{5}$, a stent technique ${ }^{13,16}$, and a sleeve anastomosis technique ${ }^{12,21-23}$. While the technique for rat OLT is still not standardized today, the arterialized model has been increasingly favored in terms of its physiological superiority $8,12,13,14$. Among the abovementioned techniques, a stent technique that was simple and fast to perform was reported by Lehmann et al. ${ }^{16}$ in 2005 . The study showed excellent results: no occlusion rate was observed in the reconstructed hepatic artery at $8 \mathrm{hr}, 24 \mathrm{hr}$, and 6 months after reperfusion. We therefore adopted this technique for hepatic arterialization.

We perform a hand-sewn anastomosis for the reconstruction of the SHVC and IHVC. This method provides the anastomotic site with an optimal physiological condition, which leads to the reduced incidence of thrombosis ${ }^{8}$, and is the best microsurgery simulation and training for surgeons. In addition, the anastomosis can be possible even with short vessel stumps. Concerning the anastomosis of IHVC, this method does not require a long IHVC on the graft side compared with the cuff anastomosis technique. Therefore, when the donor renal vein is dissected to make the graft IHVC long, this method is applicable to transplantation of a small graft that necessitates a long IHVC, such as a $30 \%$ graft that consists of right lateral and caudate lobes with a short intrahepatic vena cava without SHVC ${ }^{2}$.

Regarding the techniques of liver resection in rats, to date several methods have been reported; the two major techniques are the classical mass ligature technique and the vessel-oriented technique ${ }^{24}$. We perform the classical ligature technique for $50 \%$ liver resection ${ }^{15}$, but under a surgical microscope to make the procedure finer, and to avoid damage to the remaining lobes and structures.

We described the representative results from the recipient rats in our model; the rats survived during the 7-day observation period without apparent complications. The model can be modified for different purposes of experiments by choosing the different settings, such as prolonged cold storage, prolonged warm ischemia that includes donation after cardiac death, and the usage of smaller liver grafts or grafts from experimental models of liver injury or diseases.

In our experience, there are three key factors throughout the procedures that can affect the survival after transplantation, the most reliable parameter for outcomes of rat OLT: the amount of blood loss; the operation time, especially clamping time of the portal vein and IHVC; and the adequacy of the reconstruction of each vessel, which could result in stenosis, thrombosis, or bleeding. In a training period of this model, most of the failures could probably be related to those factors. In this video article, we present step-by-step instructions for the surgical procedures for our rat model of partial OLT with hepatic arterial reconstruction. While a rat model of OLT is complicated and requires advanced microsurgical skills, this article provides plenty of practical information, which should serve as a good guide for the training and learning of this model. Learning this model efficiently is particularly important for shortening the learning period, reducing the number of animals and costs needed for practice, and later reproducing reliable results in experiments. This is in line with the 3 Rs concept (replacement, reduction, and refinement) of animal experiments, which was postulated by Russell and Burch in $1959^{25}$.

\section{Disclosures}

We have no conflicting interests to disclose. The rats are housed under specific-pathogen-free conditions according to the guidelines of the Federation for Laboratory Animal Science Associations (FELASA). All experiments were conducted in accordance with the German federal law 
regarding the protection of animals and "Guide for the Care and Use of Laboratory Animals" (National Institutes of Health publication No. 86-23, revised 1985).

\section{Acknowledgements}

The authors thank Pascal Paschenda and Mareike Schulz for their technical assistance.

\section{References}

1. Puhl, G., et al. Low viscosity histidine-tryptophan-ketoglutarate graft flush improves subsequent extended cold storage in University of Wisconsin solution in an extracorporeal rat liver perfusion and rat liver transplantation model. Liver Transpl. 12, 1841-1849 (2006).

2. Yagi, S., et al. Improved Preservation and Microcirculation with POLYSOL After Partial Liver Transplantation in Rats. J Surg Res. 167, e375-383 (2011).

3. Engemann, R., Ulrichs, K., Thiede, A., Muller-Ruchholtz, W., \& Hamelmann, H. Value of a physiological liver transplant model in rats. Induction of specific graft tolerance in a fully allogeneic strain combination. Transplantation. 33, 566-568 (1982).

4. Sumimoto, R., Shinomiya, T., \& Yamaguchi, A. Influence of hepatic arterial blood flow in rats with liver transplants. Examination of donor liverderived serum class I MHC antigen in rats with liver transplants with or without hepatic arterial reconstruction. Transplantation. 51, 1138-1139 (1991).

5. Chaland, P., et al. Orthotopic liver transplantation with hepatic artery anastomoses. Hemodynamics and response to hemorrhage in conscious rats. Transplantation. 49, 675-678 (1990).

6. Imamura, H., Rocheleau, B., Cote, J., \& Huet, P.M. Long-term consequence of rat orthotopic liver transplantation with and without hepatic arterial reconstruction: a clinical, pathological, and hemodynamic study. Hepatology. 26, 198-205 (1997).

7. Zhong, Z., Theruvath, T.P., Currin, R.T., Waldmeier, P.C., \& Lemasters, J.J. NIM811, a mitochondrial permeability transition inhibitor, prevents mitochondrial depolarization in small-for-size rat liver grafts. Am. J. Transplant. 7, 1103-1111 (2007).

8. Spiegel, H.U. \& Palmes, D. Surgical techniques of orthotopic rat liver transplantation. J. Invest. Surg. 11, 83-96 (1998).

9. Lee, S., Charters, A.C., Chandler, J.G., \& Orloff, M.J. A technique for orthotopic liver transplantation in the rat. Transplantation. 16, 664-669 (1973).

10. Lee, S., Charters, A.C., 3rd, \& Orloff, M.J. Simplified technic for orthotopic liver transplantation in the rat. Am. J. Surg. 130, 38-40 (1975).

11. Kamada, N. \& Calne, R.Y. Orthotopic liver transplantation in the rat. Technique using cuff for portal vein anastomosis and biliary drainage. Transplantation. 28, 47-50 (1979).

12. Howden, B., Jablonski, P., Grossman, H., \& Marshall, V.C. The importance of the hepatic artery in rat liver transplantation. Transplantation 47, 428-431 (1989).

13. Gao, W., Lemasters, J.J., \& Thurman, R.G. Development of a new method for hepatic rearterialization in rat orthotopic liver transplantation. Reduction of liver injury and improvement of surgical outcome by arterialization. Transplantation. 56, 19-24 (1993).

14. Zhao, D., Zimmermann, A. \& Wheatley, A.M. Morphometry of the liver after liver transplantation in the rat: significance of an intact arterial supply. Hepatology. 17, 310-317 (1993).

15. Omura, T., Ascher, N.L., \& Emond, J.C. Fifty-percent partial liver transplantation in the rat. Transplantation. 62, 292-293 (1996).

16. Lehmann, T.G., Bunzendahl, H., Langrehr, J.M., \& Neuhaus, P. Arterial reconstruction in rat liver transplantation--development of a new tubing technique of the common hepatic artery. Transpl. Int. 18, 56-64 (2005).

17. Reck, T., et al. Impact of arterialization on hepatic oxygen supply, tissue energy phosphates, and outcome after liver transplantation in the rat. Transplantation. 62, 582-587 (1996).

18. Hasuike, Y., et al. A simple method for orthotopic liver transplantation with arterial reconstruction in rats. Transplantation. 45, 830-832 (1988).

19. Steffen, R., Ferguson, D.M., \& Krom, R.A. A new method for orthotopic rat liver transplantation with arterial cuff anastomosis to the recipient common hepatic artery. Transplantation. 48, 166-168 (1989).

20. Knoop, M., Bachmann, S., Keck, H., Steffen, R., \& Neuhaus, P. Experience with cuff rearterialization in 600 orthotopic liver grafts in the rat. Am. J. Surg. 167, 360-363, [pii] 0002-9610(94)90215-1 (1994).

21. Hickman, R., Engelbrecht, G.H., \& Duminy, F.J. A technique for liver transplantation in the rat. Transplantation. 48, 1080 (1989).

22. Liu, T., Freise, C.E., Ferrell, L., Ascher, N.L., \& Roberts, J.P. A modified vascular "sleeve" anastomosis for rearterialization in orthotopic liver transplantation in rats. Transplantation. 54, 179-180 (1992).

23. Li, J., et al. Modified sleeve anastomosis for reconstruction of the hepatic artery in rat liver transplantation. Microsurgery. 22, 62-68 (2002).

24. Martins, P.N., Theruvath, T.P., \& Neuhaus, P. Rodent models of partial hepatectomies. Liver Int. 28, 3-11 (2008).

25. Russell, W.M.S. \& Burch, R.L. The Principles of Humane Experimental Technique., Methuen \& Co. Ltd., London, reprinted by UFAW, 1992, (1959). 